\title{
Magnetic structure of $\mathrm{La}_{8} \mathrm{Cu}_{7} \mathrm{O}_{19}$
}

\author{
K. Prokeš, ${ }^{1}$ E. Ressouche, ${ }^{2}$ A. Mohan, ${ }^{3}$ A. U. B. Wolter, ${ }^{3}$ B. Büchner,,${ }^{3,4}$ and C. Hess ${ }^{3,4}$ \\ ${ }^{1}$ Helmholtz-Zentrum Berlin für Materialien und Energie, EM-AQM, Hahn-Meitner Platz 1, 14109 Berlin, Germany \\ ${ }^{2}$ SPSMS, UMR-E CEA/UJF-Grenoble 1, INAC, Grenoble, 38054, France \\ ${ }^{3}$ Leibniz-Institute for Solid State and Materials Research, IFW-Dresden, 01171 Dresden, Germany \\ ${ }^{4}$ Center for Transport and Devices, Technische Universität Dresden, 01069 Dresden, Germany
}

(Received 22 September 2014; revised manuscript received 13 December 2016; published 5 January 2017)

\begin{abstract}
The magnetic structure of the five-leg ladder compound $\mathrm{La}_{8} \mathrm{Cu}_{7} \mathrm{O}_{19}$ has been determined using single crystalline neutron diffraction. The material orders antiferromagnetically with the propagation vector $q=\left(\frac{1}{2}, \frac{1}{2}, 0\right)$ below $T_{N}=102.5(3) \mathrm{K}$. Above this temperature the magnetic susceptibility corresponds indeed to that of a five-leg $S=1 / 2$ spin ladder, assuming isotropic couplings along the rungs and the legs $\left[J / k_{B}=J^{\prime} / k_{B}=194(3) \mathrm{K}\right]$. Crystallographically, the $\mathrm{Cu}$ magnetic moments divide into two subsystems, depending on the environment. Moments situated at centers of octahedra built up by oxygens orient along the $b$ axis and couple to neighboring moments antiferromagnetically, whereas those creating a complicated ribbon structure possess all three Cartesian components. All $\mathrm{Cu}$ moments are found to have magnitudes between 0.69 and $1.32 \mu_{B}$. Thus, it is seen that the five-leg ladder $\mathrm{La}_{8} \mathrm{Cu}_{7} \mathrm{O}_{19}$ exhibits long-range antiferromagnetic ordering and possesses a rather complicated magnetic structure which has a noncollinear character.
\end{abstract}

DOI: 10.1103/PhysRevB.95.024405

\section{INTRODUCTION}

Studies of quantum magnetism in low-dimensional systems is a successful example for a fruitful interplay between theory and experiment. Often, excellent agreement between theoretical predictions and experiments has been found as far as ground-state properties, excitation spectra, thermodynamic, or optical properties are concerned [1]. Substantial progress has been made in past years, but the understanding is still incomplete, especially for systems involving many coupled degrees of freedom such as spins, orbitals, and phonons. Spin-ladder systems that exist in many different versions differing in the number of ladder legs often show very interesting transport, and quantum magnetic properties [2,3]. For instance, for the spin-1/2 antiferromagnetic Heisenberg spin ladder with isotropic coupling, increasing the number $l$ of the legs describes the crossover from the $l=1$ spin chain with a quantum-disordered ground state to the two-dimensional Heisenberg antiferromagnet on a square lattice $(l \rightarrow \infty)$ with a long-range ordered ground state. The crossover is discontinuous as the ground state properties alternate upon changing $l[1,2,4]$. For $l=$ odd, the spin-spin correlation decays quasialgebraically and the excitation spectrum is gapless. In contrast, for $l=$ even, the ground state is always a singlet state with exponentially decaying spin-spin correlations, connected with a gapped excitation spectrum. Interestingly, ladders with $l=2$ bear a potential to become superconducting under light hole doping, and thus are considered toy models for high-temperature superconductivity [4,5].

$\mathrm{La}_{8} \mathrm{Cu}_{7} \mathrm{O}_{19}$ is the $n=3$ member of the homologous series $\mathrm{La}_{4+4 n} \mathrm{Cu}_{8+2 n} \mathrm{O}_{14+8 n}$ that was discovered by Cava et al. [6] more than 20 years ago and is claimed to realize a five-leg ladder spin model [7]. $\mathrm{La}_{8} \mathrm{Cu}_{7} \mathrm{O}_{19}$ is in its as-prepared form semiconducting [8]. The conductivity can be improved by a heat treatment in an oxygen atmosphere [8,9]. Although superconductivity has not yet been found, the compound's close relation to high-temperature superconductors further motivates interest in this material.
$\mathrm{La}_{8} \mathrm{Cu}_{7} \mathrm{O}_{19}$ is reported to order antiferromagnetically (AF) below $103 \mathrm{~K}$ [10], indicative of significant three-dimensional exchange interactions. However, bulk susceptibility measurements by Cava et al. on polycrystalline $\mathrm{La}_{8} \mathrm{Cu}_{7} \mathrm{O}_{19}$ does not show any visible anomaly at this temperature [10]. To the best of our knowledge, no detailed information about the magnitude of magnetic moments involved and their spatial distribution is available in the literature. Early polarized neutron scattering data on $\mathrm{La}_{8} \mathrm{Cu}_{7} \mathrm{O}_{19}$ [10] only suggest a complicated nonlinear arrangement of $\mathrm{Cu}$ spins into two different subsystems, depending on the environment, and that the propagation vector is $q=\left(\frac{1}{2}, \frac{1}{2}, 0\right)$. The result of a nonpolarized experiment, which is briefly mentioned in the earlier work, is to our knowledge to date not reported. This situation prompted us to perform a new neutron diffraction experiment on a $\mathrm{La}_{8} \mathrm{Cu}_{7} \mathrm{O}_{19}$ single crystal.

\section{EXPERIMENTAL}

Single crystalline $\mathrm{La}_{8} \mathrm{Cu}_{7} \mathrm{O}_{19}$ was grown by the travelingsolvent floating-zone method using the four mirror image furnace by Crystal Systems Inc (CSI). $99.99 \%$ pure $\mathrm{La}_{2} \mathrm{O}_{3}$ and $\mathrm{CuO}$ were used as starting chemicals. The details regarding the crystal growth are published elsewhere [11].

The temperature dependence of the static magnetic susceptibility $\chi=M / H$, where $H$ denotes the applied magnetic field, was measured in the temperature range $2 \mathrm{~K}$ to $400 \mathrm{~K}$ using a $7 \mathrm{~T}$ Quantum Design SQUID-VSM by applying a field along all three principal crystallographic axes. The neutron diffraction experiment was performed on the D23 diffractometer at the Institut Laue Langevin using a standard cryostat capable of reaching temperatures down to $1.7 \mathrm{~K}$. A $\lambda / 2$ filter implies a residual higher-order wavelength contamination at a level of less than $10^{-4}$. The sample, in a form of a semicylinder with a diameter of about $3 \mathrm{~mm}$ and a length of $5 \mathrm{~mm}$, was attached by means of a dental glue to an aluminium holder with its $b$ axis along the rotational axis of the diffractometer. This geometry restricts the reachable 
number of reflections with respect to the $b$ direction, however, the accessible reflections are better resolved. We have also recorded some data with a smaller sample in a form of a cuboid with dimensions $2 \times 2 \times 2 \mathrm{~mm}^{3}$. Resulting data sets were combined. The D23 diffractometer is equipped with a conventional ${ }^{3} \mathrm{He}$ single detector providing a high detection efficiency of the diffracted intensity as the sample is rocked over a specified angular range $(\omega)$. Nuclear and magnetic reflections were collected with two neutron wavelengths of $\lambda=1.27 \AA$ and $\lambda=2.37 \AA$ that gave us the flexibility to collect a large number of nuclear reflections concentrating on the crystal structure determination and then to collect magnetic reflections that are of much weaker intensities with the longer wavelength. Each nuclear reflection was measured typically for about four minutes. To identify the magnetic signal we have performed wide reciprocal scans along symmetrical directions that included integer and half-integer directions. Detected magnetic reflections, due to their lower intensity, were subsequently measured using rocking scans four times longer than nuclear ones. Nuclear and magnetic reflections were collected at $10 \mathrm{~K}$ and at $115 \mathrm{~K}$ that is above the proposed magnetic phase transition reported in the literature [10] around $103 \mathrm{~K}$. We also followed the intensities of representative nuclear and magnetic reflections as a function of temperature between 10 and $130 \mathrm{~K}$. In this case, the measurement time was shorter by a factor of two.

\section{RESULTS}

\section{Crystal structure}

The structure of the unit cell of this compound is shown in Fig. 1. The $\mathrm{Cu}$ atoms reside in two different environments, namely in the center of $\mathrm{CuO}_{4}$ tetrahedra $(\mathrm{Cu} 3,4)$ forming the outer ladder legs and complex $\mathrm{CuO}_{2}$ ribbons, respectively, and at centers of $\mathrm{CuO}_{6}$ octahedra forming the rungs and inner legs of the ladder $(\mathrm{Cu} 1$ and $\mathrm{Cu} 2)$ [12]. Adjacent ladder planes are bridged by edge-sharing $\mathrm{CuO}_{4}$ tetrahedra along the $a$ axis. The rungs of the ladder run parallel to the [101] direction, and the legs of the ladder run along the $b$ axis ([010]).

Before performing neutron diffraction experiments at low temperatures, the samples were oriented using Laue backscattering. It has been found that the large sample has

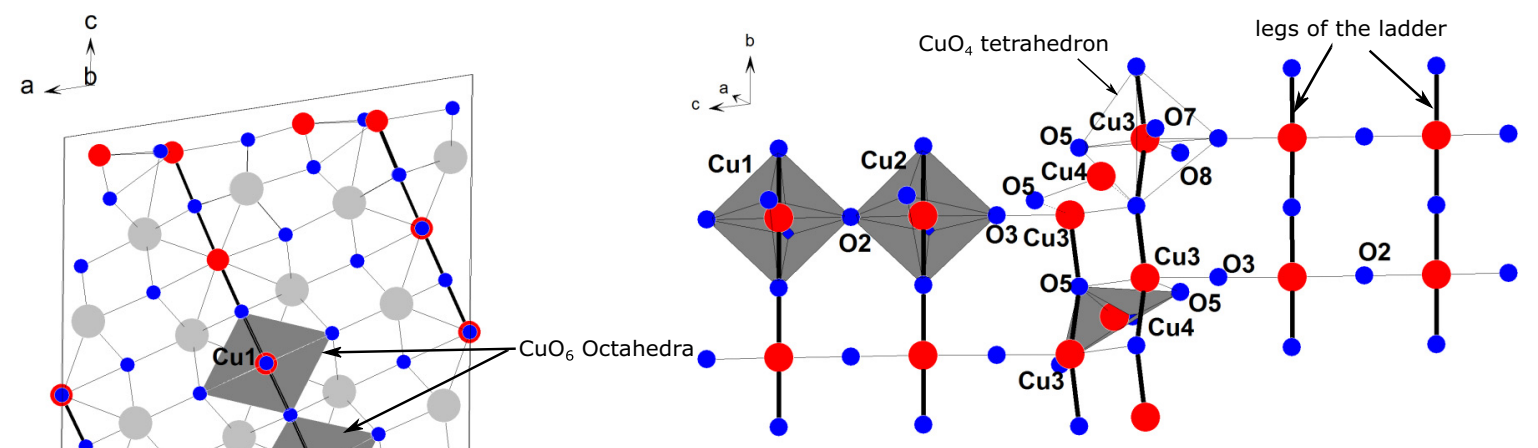

(b)

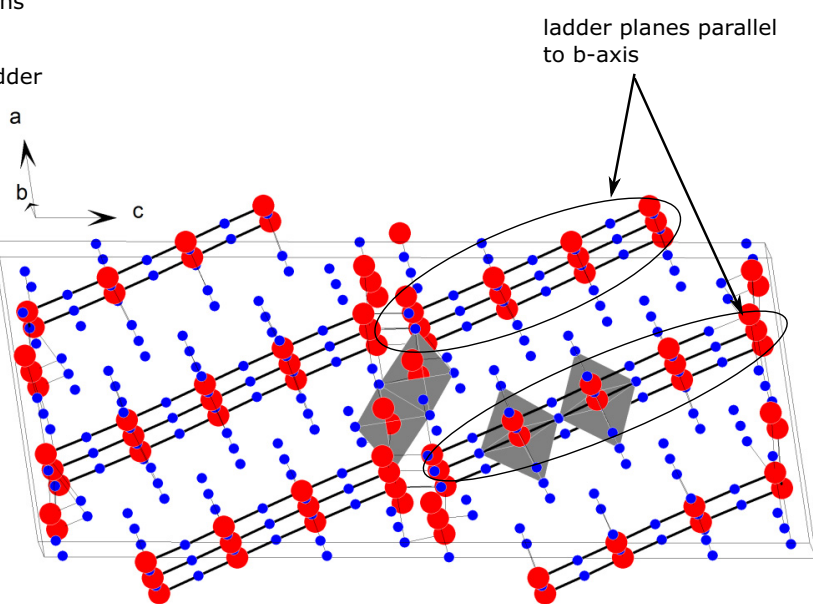

(a)

(c)

FIG. 1. Crystal structure of $\mathrm{La}_{8} \mathrm{Cu}_{7} \mathrm{O}_{19}$. Smallest spheres (blue): O, largest spheres (gray): La, medium spheres (blue): Cu. (a) The crystallographically inequivalent $\mathrm{Cu}$ atoms are marked. The rungs of the adjacent five-leg ladders are connected via the ribbon structure formed by $\mathrm{Cu} 3$ and $\mathrm{Cu} 4$ atoms. $\mathrm{Cu}$ atoms are distributed over four inequivalent crystallographic sites and possess two different environments, $\mathrm{CuO}_{6}$ octahedrons $(\mathrm{Cu} 1,2)$ and $\mathrm{CuO}_{4}$ tetrahedrons $(\mathrm{Cu} 3,4)$. The crystallographically inequivalent oxygen atoms $(\mathrm{O} 2,3,5,7,8)$ are marked. $(\mathrm{b})$ The joining of two five-leg ladder units is depicted, where one can see $\mathrm{Cu} 3$ and $\mathrm{Cu} 4$ atoms forming a complex ribbonlike structure. $\mathrm{The}^{\mathrm{CuO}}{ }_{4}$ tetrahedron around the $\mathrm{Cu} 3$ atoms formed by $\mathrm{O}$ atoms $(\mathrm{O} 5,3)$ is depicted. The O5,7,8 oxygen atoms are situated out of the plane of the page. (c) A-three dimensional view of the unit cell where one can see the ladder planes. In (b) and (c) only $\mathrm{Cu}$ and $\mathrm{O}$ atoms are shown for clarity. 

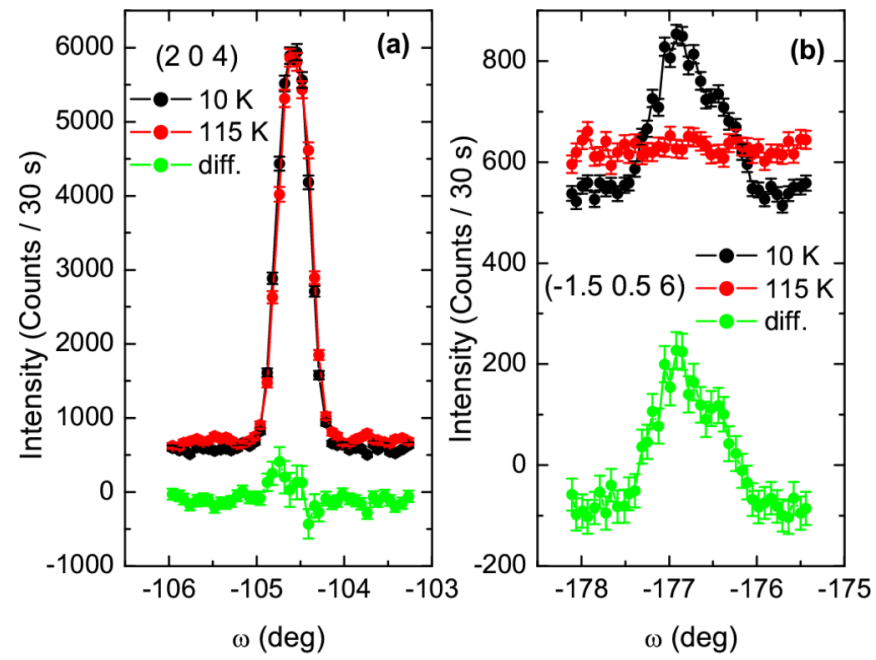

FIG. 2. Typical rocking curves of the nuclear Bragg reflection (2 04 ) at $10 \mathrm{~K}$ and $115 \mathrm{~K}$, together with the difference between them (a) and of the magnetic Bragg reflection $\left(-\frac{3}{2} \frac{1}{2} 6\right)$ measured on the large sample for identical conditions (b). All measurements were scaled to the same monitor.

a good quality with the width of the Bragg reflections limited by the resolution function of the diffractometer, while the smaller sample consists of two main grains, split by about $1.2^{\circ}$. Afterwards, the samples were cooled down to low temperatures. As an example, we show in Fig. 2(a), a rocking curve of the (2 04$)$ nuclear reflection measured on the larger sample at $10 \mathrm{~K}$, well below the $T_{N}$, and at $115 \mathrm{~K}$, that is above the proposed magnetic phase transition, together with the difference. No extra diffracted intensity is found at low temperatures at the top of nuclear reflections. Also, we have not obtained any evidence of a structural distortion upon cooling. The knowledge of structural details is a prerequisite for the magnetic structure determination. In total, we have measured on the larger sample 120 and on the smaller 51 unique reflections serving as a basis to determine the scaling factor and extinction correction needed for a correct magnetic structure and magnetic moment determination.

In order to refine the structure parameters of $\mathrm{La}_{8} \mathrm{Cu}_{7} \mathrm{O}_{19}$ the data were normalized to the same monitor and fit to a structural model known from the literature [7] using the computer code FULLPROF which is a part of a larger package Winplotr [13]. The incorporated secondary type extinction correction that describes the decrease of reflection intensities due to an angular distribution of large mosaic blocks has been applied and found to be rather weak. The tabulated values of the scattering length of elements present in the sample were used in the refinement.

Because of a large number of free structural parameters we have restricted ourselves to the use of isotropic temperature factors and stoichiometric occupations of all the elements. The plot of observed versus calculated squared structure factors after the use of extinction and correction for the Lorentz geometrical factor is shown in Fig. 3. As can be seen, a satisfactory agreement (all the data fall on a single straight line) is obtained. Structural parameters that are listed in Table I are in very good agreement with literature values $[6,7,9,14]$ except for the $y$ positional parameters. These are determined

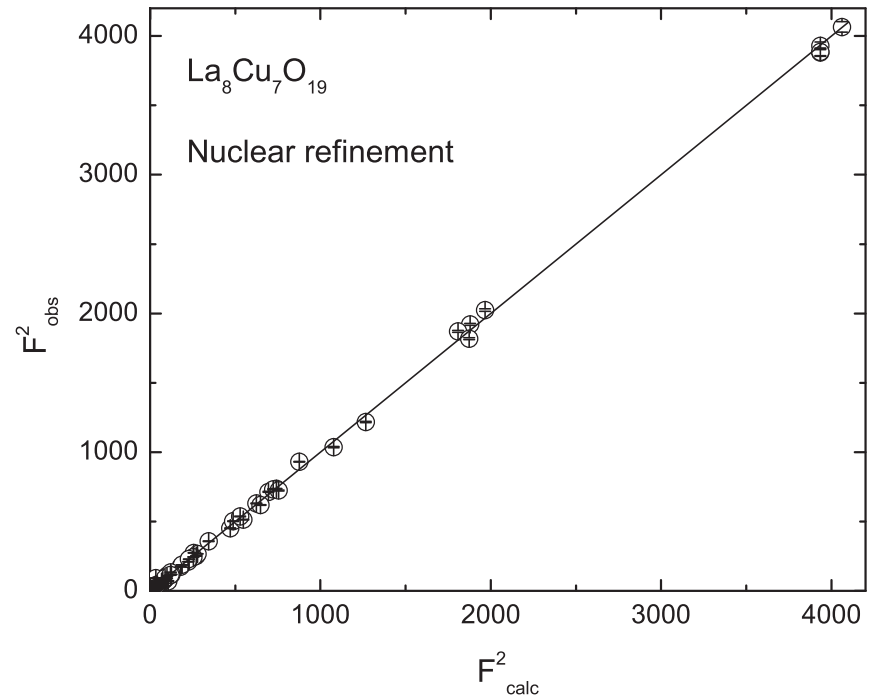

FIG. 3. Plot of the observed versus calculated squared nuclear structure factors collected on the large $\mathrm{La}_{8} \mathrm{Cu}_{7} \mathrm{O}_{19}$ single crystal after correction for the extinction and Lorentz factor, and refinement using the model shown in Fig. 6.

with a lesser degree of precision due to a limited number of accessible $(h k l)$ reflections with a nonzero $k$ index. Also a

TABLE I. Structural parameters of $\mathrm{La}_{8} \mathrm{Cu}_{7} \mathrm{O}_{19}$ as determined from neutron single crystal data collected with $\lambda=1.27 \AA$ at $10 \mathrm{~K}$.

\begin{tabular}{|c|c|c|c|c|}
\hline $\mathrm{La}_{8} \mathrm{Cu}_{7} \mathrm{O}_{19}$ & $T=10 \mathrm{~K}$ & Space group: & $C 2 / c$ & \\
\hline$a(\AA)$ & $13.831(2)$ & & & \\
\hline$b(\AA)$ & $3.758(8)$ & & & \\
\hline$c(\AA)$ & $34.592(6)$ & & & \\
\hline$\beta(\operatorname{deg})$ & $99.30(4)$ & & & \\
\hline$V\left(\AA^{3}\right)$ & 1774.35 & & & \\
\hline Observed refl. & 120 & & & \\
\hline $\mathrm{R}_{f}^{2}(\%)$ & 2.83 & & & \\
\hline $\mathrm{R}_{f}(\%)$ & 3.77 & & & \\
\hline \multicolumn{5}{|c|}{ Atomic positional parameters: } \\
\hline Label (site) & $x$ & $y$ & $z$ & $B\left(\AA^{2}\right)$ \\
\hline La1(8f) & $0.4363(3)$ & $0.9696(15)$ & $0.3178(1)$ & $0.28(3)$ \\
\hline $\mathrm{La} 2(8 f)$ & $0.2996(2)$ & $0.4651(18)$ & $0.3933(1)$ & La1 \\
\hline $\operatorname{La3}(8 f)$ & $0.5496(3)$ & $0.9997(16)$ & $0.4228(1)$ & La1 \\
\hline $\mathrm{La} 4(8 \mathrm{f})$ & $0.1816(3)$ & $0.4747(15)$ & $0.2877(1)$ & La1 \\
\hline $\mathrm{Cu} 1(4 \mathrm{e})$ & 0 & $-0.049(5)$ & $\frac{1}{4}$ & $0.25(2)$ \\
\hline $\mathrm{Cu} 2(8 \mathrm{f})$ & $0.6192(5)$ & $0.5185(28)$ & $0.3582(2)$ & $0.27(3)$ \\
\hline $\mathrm{Cu} 3(8 \mathrm{f})$ & $0.7329(4)$ & $0.5090(45)$ & $0.4691(2)$ & $\mathrm{Cu} 2$ \\
\hline $\mathrm{Cu} 4(8 \mathrm{f})$ & $0.5878(5)$ & $0.3735(16)$ & $0.5243(2)$ & $\mathrm{Cu} 2$ \\
\hline $\mathrm{O} 1(4 \mathrm{e})$ & 0 & $0.426(3)$ & $\frac{1}{4}$ & $0.27(3)$ \\
\hline $\mathrm{O} 2(8 \mathrm{f})$ & $0.5616(5)$ & $0.4812(21)$ & $0.3040(2)$ & $0.27(3)$ \\
\hline O3(8f) & $0.6789(4)$ & $0.4906(23)$ & $0.4138(2)$ & $\mathrm{O} 2$ \\
\hline O4(8f) & $0.6202(4)$ & $0.0147(23)$ & $0.3586(2)$ & $\mathrm{O} 2$ \\
\hline O5(8f) & $0.7345(4)$ & $0.4987(60)$ & $0.5271(2)$ & $\mathrm{O} 2$ \\
\hline O6(8f) & $0.7770(4)$ & $0.4737(22)$ & $0.3366(2)$ & $\mathrm{O} 2$ \\
\hline O7(8f) & $0.6129(5)$ & $0.0395(24)$ & $0.5680(2)$ & $\mathrm{O} 2$ \\
\hline O8(8f) & $0.5472(5)$ & $0.5220(23)$ & $0.4699(2)$ & $\mathrm{O} 2$ \\
\hline O9(8f) & $0.4553(4)$ & $0.4565(22)$ & $0.3716(2)$ & $\mathrm{O} 2$ \\
\hline O10(8f) & $0.3383(4)$ & $0.4718(19)$ & $0.2689(2)$ & $\mathrm{O} 2$ \\
\hline
\end{tabular}




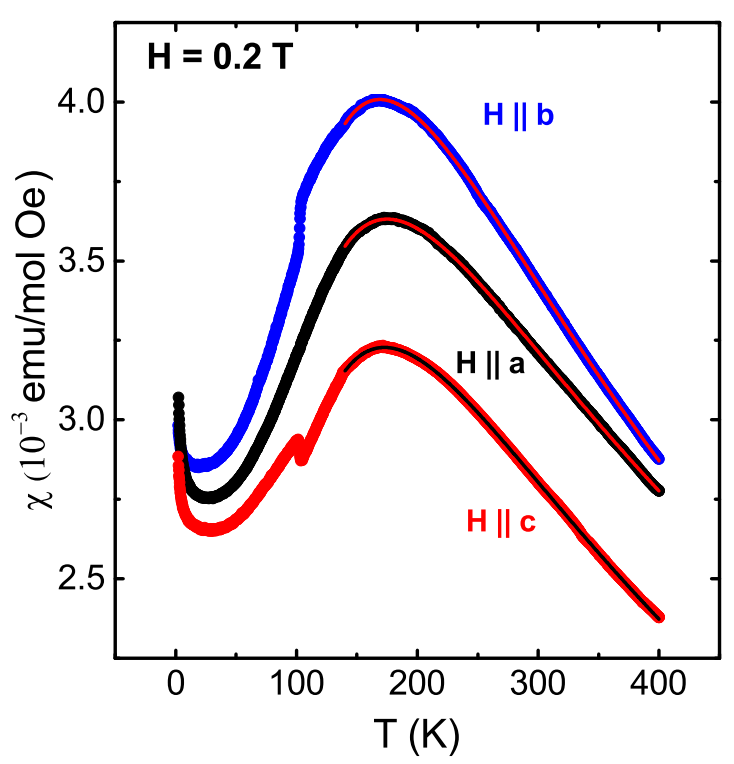

FIG. 4. Temperature dependence of the magnetic susceptibility $\chi=M / H$ of $\mathrm{La}_{8} \mathrm{Cu}_{7} \mathrm{O}_{19}$ measured with field of $0.2 \mathrm{~T}$ applied along all three principal directions $a, b$, and $c$. Full lines through the points above $140 \mathrm{~K}$ are the best fits to the model described in the main text.

satisfactory agreement is found for the structural data obtained between the two crystals. This gives us confidence that the scaling factor and the extinction parameter inferred from the nuclear fits can be used in the determination of the magnetic structure.

\section{Bulk magnetic susceptibility}

In Fig. 4 we show the temperature dependence of the magnetic susceptibility $\chi=M / H$ measured in a wide temperature range from $2-400 \mathrm{~K}$ with a field of $0.2 \mathrm{~T}$ applied along the three principal crystallographic directions. While the qualitative temperature dependence of the susceptibility is very similar for all field directions, there are clear differences in the magnitude, where the susceptibility along the $b$ axis (direction of the ladder legs) is found to be the highest and along the $c$ axis the smallest. Upon cooling below $400 \mathrm{~K}, \chi$ first shows a paramagnetic upturn but then develops a broad maximum at $T \approx 180 \mathrm{~K}$. Such a maximum is characteristic for quasione-dimensional AF quantum magnets with $S=1 / 2$, typical for odd-leg spin ladders $[3,14,15]$, and has been previously observed also for $\mathrm{La}_{8} \mathrm{Cu}_{7} \mathrm{O}_{19}$ [6,14]. Further reducing the temperature leads first to a strong decrease, which is followed by a significant upturn at even lower temperature $(T \lesssim 30 \mathrm{~K})$. We attribute the latter to a presence of a minor paramagnetic impurity and thus ignore it in the further analysis at higher temperatures.

A closer inspection of the data reveals that $\chi$ exhibits a clear anomaly around $103 \mathrm{~K}$. It manifests itself as a sudden increase (decrease) of $\chi(T)$ with the magnetic field along the $c$ axis ( $b$ axis) and is absent for a magnetic field along $a$. The temperature dependence of the magnetization was also measured at higher magnetic fields up to $7 \mathrm{~T}$, but there was no effect on the transition temperature. The observed anisotropy in the magnetic response suggests a spin anisotropy in the system. Indeed, as we will see below, the origin of this anomaly at $103 \mathrm{~K}$ is magnetic in nature-a long-range antiferromagnetic order appears in the system below this temperature. From the magnetic point of view the system becomes three dimensional. This in turn suggests that a significant exchange coupling exists between the ladder structures of the material.

In order to further analyze the observed magnetic response, we approximate $\chi(T)$ with respect to expected characteristics of a five-leg $S=1 / 2$ spin ladder with isotropic ladder and rung coupling $J^{\prime}=J$ for $T \geqslant 140 \mathrm{~K}$. Johnston et al. have derived a parametrization of quantum Monte Carlo results of Frischmuth et al. $[15,16]$ for the susceptibility of a $S=1 / 2$ five-leg ladder as

$$
\chi(T)=\chi_{0}+\frac{C}{T} * \frac{1-N_{1} x+N_{2} x^{2}+N_{3} x^{3}}{1+D_{1} x+D_{2} x^{2}+D_{3} x^{3}+D_{4} x^{4}},
$$

where $\chi_{0}$ accounts for temperature independent parts of the susceptibility, $C=f * \frac{N_{A} g^{2} \mu_{B}^{2}}{4 k_{B}}$ with $f$ measuring the contributing number of spins per formula unit (up to seven), Avogadro's constant $N_{A}$, the g-factor $g$, the Bohr magneton $\mu_{B}$, and $k_{B}$ the Boltzmann constant. $N_{1}=0.2732853, N_{2}=$ $0.09333487, \quad N_{3}=0.006660300, D_{1}=0.6267147, \quad D_{2}=$ $0.3077097, D_{3}=0.04438012, D_{4}=0.07488932$, and $x=$ $\left(J / k_{B} T\right)$ with the exchange constant $J[15]$.

The best fit is shown in Fig. 4 by full lines through the experimental points, where we obtained $J / k_{B}=194$ (3) $\mathrm{K}$, $C=1.97(25) \mathrm{emu} \mathrm{K} \mathrm{mol}{ }^{-1} \mathrm{Oe}^{-1}$ as average value for the three principal crystallographic directions, and where $\chi_{0}$ depends on the direction and is of the order of $-5(3) \times$ $10^{-4} \mathrm{emu} /(\mathrm{mol} \mathrm{Oe})^{-1}$. Allowing for different $J$ values along the different principal axes, the highest value is found for the $a$ direction $\left(J_{a} / k_{B}=197 \mathrm{~K}\right)$ and the smallest one for the $b$ direction $\left(J_{b} / k_{B}=191 \mathrm{~K}\right)$, suggesting a slight exchange anisotropy. If we assume realistic values for the $g$ factors ( $\left.g_{\|}=2.25, g_{\perp}=2.05\right)$, from the fitted $C$ values for the three directions one can deduce that about $5 \mathrm{Cu}$ spins participate in the magnetic response. Thus, the susceptibility data appear well consistent with expectations for a five-leg $S=1 / 2$ spin ladder. We stress that the agreement is twofold in the sense that not only the qualitative temperature dependence but also the magnitude of the susceptibility fit very well [17]. There are, however, some limitations of the model. Firstly, in lack of any suitable theory for this particular case our analysis relies on theoretical results for a spin ladder with the same exchange coupling $J$ along the legs and along all rungs. It is to be expected that this situation is not perfectly fulfilled in our material. Secondly, and quite surprisingly, the value of the extracted $J$ appears by about one order of magnitude too small in view of near $180^{\circ} \mathrm{Cu}-\mathrm{O}-\mathrm{Cu}$ bonds along rungs and legs which typically yields $J \sim 1000 \ldots 2000 \mathrm{~K}$. At present we do not have an explanation for this puzzling observation. One might speculate that the off-ladder $\mathrm{Cu} 4$ sites or intraladder frustration due to next-nearest-neighbor interaction plays a crucial role here. Further, relevant interladder couplings, which have not been taken into account in the modeling, might re-normalize the intraladder coupling constant and yield an effective (smaller) value for $J$. 


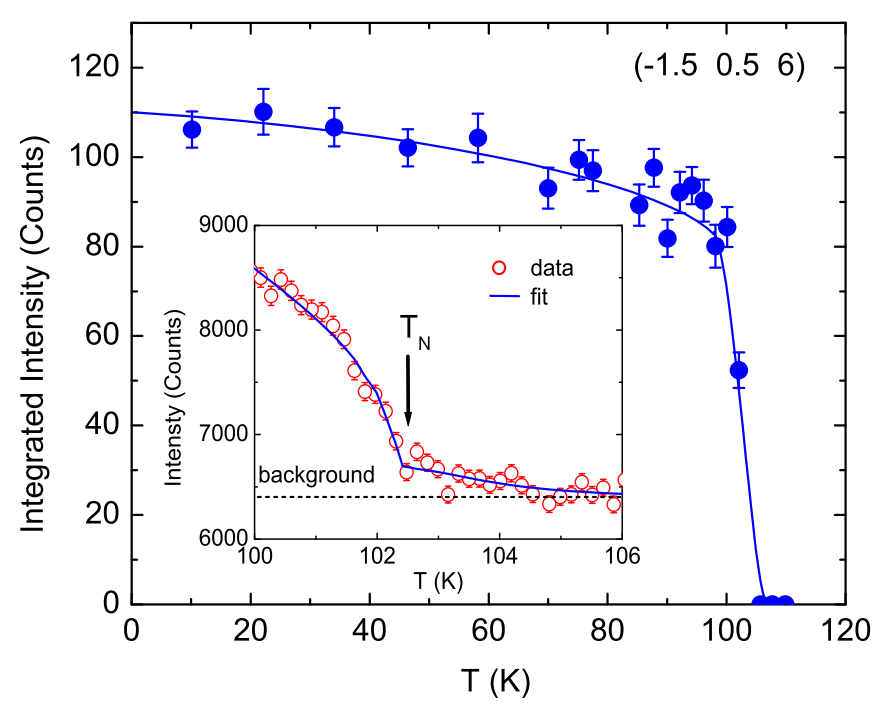

FIG. 5. Temperature dependence of the integrated intensity of the representative magnetic reflection $\left(-\frac{3}{2} \frac{1}{2} 6\right)$ measured on the large $\mathrm{La}_{8} \mathrm{Cu}_{7} \mathrm{O}_{19}$ sample in zero field with increasing temperature. In the inset we show the temperature dependence of the peak intensity of the same reflection measured in a small temperature range around the magnetic phase transition. The best fit to the formula describing the decrease of the staggered magnetization in an antiferromagnet that includes a critical scattering [19] is shown by the full blue line.

\section{Antiferromagnetic structure}

Long reciprocal scans revealed that all the Bragg reflections due to magnetic order can be indexed with a unique propagation vector $q=\left(\frac{1}{2}, \frac{1}{2}, 0\right)$. Let us note that we have selected this vector rather than vector $q^{\prime}=\left(-\frac{1}{2}, \frac{1}{2}, 0\right)$ because of intensity relations with the nuclear reflections.

In Fig. 2(b), rocking curves through a representative magnetic reflection [here $\left(\begin{array}{lll}\frac{-3}{2} & \frac{1}{2} & 6\end{array}\right)=\left(\begin{array}{lll}-2 & 0 & 6\end{array}\right)^{+q}$ ], measured on the large $\mathrm{La}_{8} \mathrm{Cu}_{7} \mathrm{O}_{19}$ sample at $10 \mathrm{~K}$ and $115 \mathrm{~K}$ are shown. While at low temperatures we observe a clear Bragg reflection (in the case of the smaller crystal with a double-peak structure that has the same separation between the two peaks as for the nuclear reflection), no intensity can be discerned at high temperature. The difference in the scattered intensity is attributed to an AF ordering. Indeed, the temperature dependence of the integrated intensity shown in Fig. 5 documents that although the intensity decreases only slowly with increasing temperature, it suddenly drops above $\approx 95 \mathrm{~K}$ and disappears above the proposed magnetic phase transition temperature. A more detailed temperature dependence of the $\left(\begin{array}{lll}-2 & 0 & 6\end{array}\right)^{+q}$ reflection is shown in the inset of Fig. 5. This plot describes the temperature dependence of the staggered magnetization in an antiferromagnet as seen by neutrons. It can be fit using, e.g., an empirical formula [18] that describes the decrease of the intensity $I(T)$ with increasing temperature with respect to the intensity in the low-temperature limit $I(0)$ according to $I(T)=I_{0}\left(1-T / T_{N}\right)^{2 \beta}$, where $\beta$ and $T_{N}$ denote the critical parameter and the magnetic phase transition temperature, respectively.

Such a fit leads above $100 \mathrm{~K}$ to a good description of the temperature dependence revealing that the magnetic phase transition occurs at $102.5(3) \mathrm{K}$ and the critical parameter $\beta=0.23(4)$. However, the observation of scattered intensity (the tail in the inset of Fig. 5) above $T_{N}$ points to a presence of critical scattering in this material. Such a critical scattering can be accounted for by a theory developed by Bruce [19] that leads to an improved description for the ordered $\mathrm{Cu}$ spins. In this case, the best fit that is shown in Fig. 5 yields the transition temperature $T_{N}=102.36(4) \mathrm{K}$ and the critical parameter $\beta=0.27(2)$. While the value of $T_{N}$ agrees well with our magnetic bulk data and other literature sources [10], the parameter $\beta$ is larger than that of Zobkalo et al. $[\beta=0.13(2)]$ [10]. For further discussion regarding the beta parameter see Sec. IV.

Since most of the magnetic reflections at low temperatures seem to be resolution limited, the magnetic order is of a longrange character. The larger width of magnetic reflections with respect to nuclear ones is due to poorer resolution as one moves above the horizontal scattering plane due to a nonzero $k$ index (Fig. 2). The apparent increase of the background in the paramagnetic state with respect to the magnetically ordered state at low temperature [as documented in Fig. 2(b)] is due to fluctuating moments above the magnetic phase transition.

Now, we turn to the determination of the AF structure in $\mathrm{La}_{8} \mathrm{Cu}_{7} \mathrm{O}_{19}$. Let us first consider the model briefly described in the work by Zobkalo et al. using polarized neutron diffraction [10]. A great portion of confusion exists concerning this model. Authors of this paper claim that $\mathrm{Cu}$ moments in octahedral coordination are oriented along the $b$ axis and coupled AF along the $c$ axis, whereas $\mathrm{Cu}$ moments within the ribbons are oriented along the $a$ axis forming ferromagnetic pairs and are coupled AF as one moves along the $a$ axis. Such a description is in our opinion ambiguous even if we assume that the authors describe the $\mathrm{Cu}$ moment coupling within one chemical unit cell. Due to a large number of atoms in the unit cell, there are several different possibilities that agree with the above mentioned description. Moreover, it does not give any information regarding the propagation along the $a$ axis for the former type of moments, and similarly, no information on the $c$ axis coupling for the latter one. Also, no information on the coupling along the $b$ axis is given. It may even suggest an interpretation that both subsystems propagate with different propagation vectors.

Therefore, our first attempt to analyze our data was within a general model that allowed for any moment direction on any site with no coupling restrictions dictated by the symmetry. This model has 84 independent parameters and the best fit converged to $\chi^{2}=6.3$. One notes that (i) the resulting magnetic structure is noncollinear, (ii) the $\mathrm{Cu}$ moments lying on the rungs, i.e., in octahedral positions, together with adjacent moments in the ribbons, are coupled antiferromagnetically as one moves along the rung direction, and (iii) the moments in these rungs tend to be oriented either along the $b$ axis or along the $a$ axis while the rest of the moments have somewhat random orientation, however, with a rather small component along the $c$ axis. Although such a fit is the most general one, it does not take into account any symmetry elements that reduce the number of independent free parameters and might modify the structure significantly.

To generate all possible magnetic structures allowed by symmetry of the crystal structure and the experimental propagation vector, we have utilized the representation analysis 
TABLE II. Possible magnetic moment couplings for the irreducible representation $\Gamma_{1}$ between $\mathrm{Cu}$ magnetic moments resulting from magnetic group theory. Symmetry requires that the moments are coupled within pairs (e.g., moments at e 1 and e 3 site). For the representation $\Gamma_{2}$ the coupling within the pairs is reversed.

\begin{tabular}{lcccccc}
\hline \hline Site & $x$ & $y$ & $z$ & $\mathrm{~m}_{x}$ & $\mathrm{~m}_{y}$ & $\mathrm{~m}_{z}$ \\
\hline $\mathrm{e} 1$ & 0 & 0.951 & 0.25 & $x_{e 1}$ & $y_{e 1}$ & $z_{e 1}$ \\
$\mathrm{e} 2$ & 0.5 & 0.451 & 0.25 & $x_{e 2}$ & $y_{e 2}$ & $z_{e 2}$ \\
$\mathrm{e} 3$ & 0 & 0.049 & 0.75 & $-x_{e 1}$ & $-y_{e 1}$ & $-z_{e 1}$ \\
$\mathrm{e} 4$ & 0.5 & 0.549 & 0.75 & $-x_{e 2}$ & $-y_{e 2}$ & $-z_{e 2}$ \\
$\mathrm{f} 11$ & 0.619 & 0.518 & 0.358 & $x_{f 11}$ & $y_{f 11}$ & $z_{f 11}$ \\
$\mathrm{f} 12$ & 0.380 & 0.518 & 0.141 & $x_{f 12}$ & $y_{f 12}$ & $z_{f 12}$ \\
$\mathrm{f} 13$ & 0.380 & 0.481 & 0.641 & $-x_{f 11}$ & $-y_{f 11}$ & $-z_{f 11}$ \\
$\mathrm{f} 14$ & 0.619 & 0.481 & 0.858 & $-x_{f 12}$ & $-y_{f 12}$ & $-z_{f 12}$ \\
$\mathrm{f} 15$ & 0.119 & 0.018 & 0.358 & $x_{f 15}$ & $y_{f 15}$ & $z_{f 15}$ \\
$\mathrm{f} 16$ & 0.880 & 0.018 & 0.141 & $x_{f 16}$ & $y_{f 16}$ & $z_{f 16}$ \\
$\mathrm{f} 17$ & 0.880 & 0.981 & 0.641 & $-x_{f 15}$ & $-y_{f 15}$ & $-z_{f 15}$ \\
$\mathrm{f} 18$ & 0.119 & 0.981 & 0.858 & $-x_{f 16}$ & $-y_{f 16}$ & $-z_{f 16}$ \\
$\mathrm{f} 21$ & 0.733 & 0.509 & 0.469 & $x_{f 21}$ & $y_{f 21}$ & $z_{f 21}$ \\
$\mathrm{f} 22$ & 0.266 & 0.509 & 0.030 & $x_{f 22}$ & $y_{f 22}$ & $z_{f 22}$ \\
$\mathrm{f} 23$ & 0.266 & 0.490 & 0.530 & $-x_{f 21}$ & $-y_{f 21}$ & $-z_{f 21}$ \\
$\mathrm{f} 24$ & 0.733 & 0.490 & 0.969 & $-x_{f 22}$ & $-y_{f 22}$ & $-z_{f 22}$ \\
$\mathrm{f} 25$ & 0.233 & 0.009 & 0.469 & $x_{f 25}$ & $y_{f 25}$ & $z_{f 25}$ \\
$\mathrm{f} 26$ & 0.766 & 0.009 & 0.030 & $x_{f 26}$ & $y_{f 26}$ & $z_{f 26}$ \\
$\mathrm{f} 27$ & 0.766 & 0.990 & 0.530 & $-x_{f 25}$ & $-y_{f 25}$ & $-z_{f 25}$ \\
$\mathrm{f} 28$ & 0.233 & 0.990 & 0.969 & $-x_{f 26}$ & $-y_{f 26}$ & $-z_{f 26}$ \\
$\mathrm{f} 31$ & 0.587 & 0.374 & 0.524 & $x_{f 31}$ & $y_{f 31}$ & $z_{f 31}$ \\
$\mathrm{f} 32$ & 0.412 & 0.374 & 0.975 & $x_{f 32}$ & $y_{f 32}$ & $z_{f 32}$ \\
$\mathrm{f} 33$ & 0.412 & 0.625 & 0.475 & $-x_{f 31}$ & $-y_{f 31}$ & $-z_{f 31}$ \\
$\mathrm{f} 34$ & 0.587 & 0.625 & 0.024 & $-x_{f 32}$ & $-y_{f 32}$ & $-z_{f 32}$ \\
$\mathrm{f} 35$ & 0.087 & 0.874 & 0.524 & $x_{f 35}$ & $y_{f 35}$ & $z_{f 35}$ \\
$\mathrm{f} 36$ & 0.912 & 0.874 & 0.975 & $x_{f 36}$ & $y_{f 36}$ & $z_{f 36}$ \\
$\mathrm{f} 37$ & 0.912 & 0.125 & 0.475 & $-x_{f 35}$ & $-y_{f 35}$ & $-z_{f 35}$ \\
$\mathrm{f} 38$ & 0.087 & 0.125 & 0.024 & $-x_{f 36}$ & $-y_{f 36}$ & $-z_{f 36}$ \\
\hline \hline & & & & & & \\
\hline
\end{tabular}

as developed by Bertaut [20] and implemented it in the computer code BasisReps [13]. The $28 \mathrm{Cu}$ atoms within the crystallographic unit cell are distributed over four inequivalent $\mathrm{Cu}$ crystallographic sites [7] denoted in Fig. 1 as $\mathrm{Cu} 1, \mathrm{Cu} 2$, $\mathrm{Cu} 3$, and $\mathrm{Cu} 4$. One of them, $\mathrm{Cu} 1$, is the $4 \mathrm{e}$ site with a local twofold axis. Consequently, we denote the four magnetic moments in this $4 \mathrm{e}$ site in the following text as e1, e2, e3, and 4 . The three remaining positions, $\mathrm{Cu} 2, \mathrm{Cu} 3$, and $\mathrm{Cu} 4$ are of the $8 f$ type (differing in positional parameters) with a local symmetry 1 , i.e., without any symmetry constraints. We denote the three groups of eight moments in the following text as $\mathrm{f} 11, \ldots, \mathrm{f} 18, \mathrm{f} 21, \ldots, \mathrm{f} 28$ and $\mathrm{f} 31, \ldots, \mathrm{f} 38$.

The calculation reveals that there are two one-dimensional irreducible representations and that all the moments are split into fourteen pairs of $\mathrm{Cu}$ moments. Moments situated in the 4 e site split in e1-e3 and e2-e4 pairs and those in the 8f site in fn1-fn3, fn2-fn4, fn5-fn7, and fn6-fn8 pairs, where $n=1,2$ and 3. Their spatial positions are given below in Table II and labeled in Fig. 6.

Moments within one unit cell are related by inversion either ferromagnetically or antiferromagnetically and are allowed to have any spatial orientation. In the irreducible representation $\Gamma_{1}, \mathrm{Cu}$ moments within the individual pairs are coupled

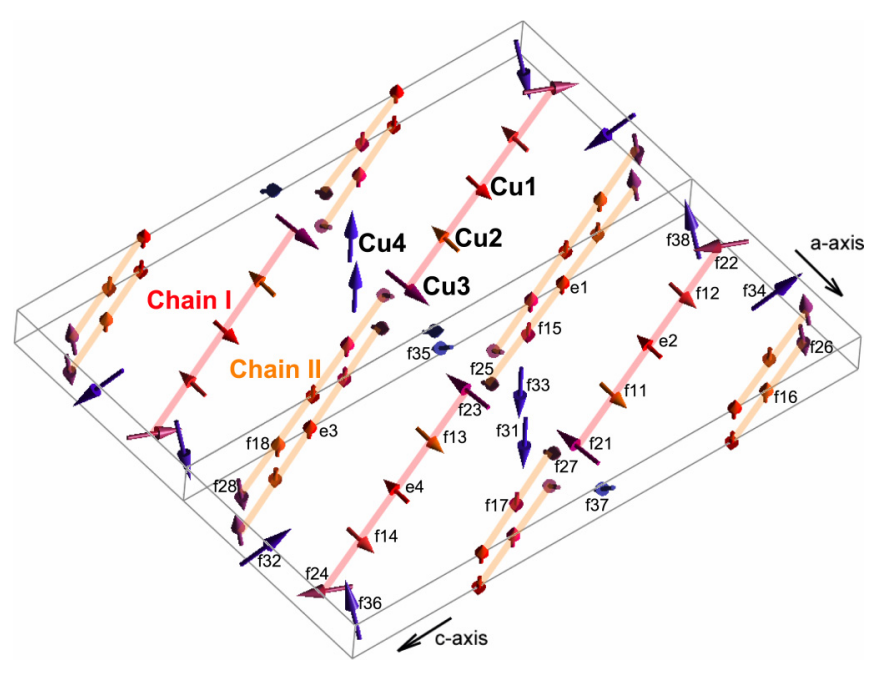

FIG. 6. Schematic representation of the AF structure of $\mathrm{La}_{8} \mathrm{Cu}_{7} \mathrm{O}_{19}$. For clarity, only the $\mathrm{Cu}$ magnetic moments are shown. Two crystallographic units $2(\mathrm{axbxc})$ are shown. The color type indicates the paired moments. Red-type colors denote moments constituting rungs, blue-type colors indicate moments situated in tetrahedral sites providing a connection between rungs. Two types of rungs with moments perpendicular to the $b$ axis and along the $b$ axis are shown by orange and red color and are denoted as Chain I and Chain II, respectively. All magnetic moments are labeled in agreement with Table II.

antiferromagnetically for all crystallographic sites. For $\Gamma_{2}$ the coupling between the pairs is reversed, i.e., ferromagnetic. The moment directions in adjacent unit cells along the $a$ axis and the $b$ axis are reversed as a consequence of the propagation vector $q=\left(\frac{1}{2}, \frac{1}{2}, 0\right)$. Along the $c$ axis they preserve their orientations. Couplings between $\mathrm{Cu}$ moments within the $\Gamma_{1}$ irreducible representation are summarized in Table II. Note the pairs of $\mathrm{Cu}$ moments.

The symmetry analysis assuming $q=\left(\frac{1}{2}, \frac{1}{2}, 0\right)$, given above, suggests that it is enough to consider instead of twentyeight magnetic moments in the unit cell only fourteen of them as independent entities. There is, however, no symmetry element that would couple moments between the individual pairs.

After fitting our data to models associated with the two irreducible representations it became clear that a better agreement is achieved for the model connected with $\Gamma_{2}$. The $\chi^{2}$ of 5.2 for this model is by a factor of four lower than for the model associated with $\Gamma_{1}$. While in the former case one arrives at $\mathrm{Cu}$ moments $\mu_{C u}$ that are on all sites rather similar, ranging from $\approx 0.7$ to $1.3 \mu_{B}$, the latter solution gives a much larger span from 0.3 to $2.4 \mu_{B}$. All moments have also much larger error bars in the latter case. Both fits have a common feature that solutions are noncollinear with moments having all three components, the $c$ axis component being rather small. Furthermore, moments within the rungs (see Fig. 6) are clearly coupled antiferromagnetically. Their refined $\phi$ values (angle with respect to the $a$ axis) suggest that they split into two types of chains with one chain having moments oriented along the $b$ axis and the other one perpendicular to it. Within these chains moments tend to be collinear. 
TABLE III. Refined magnetic structure parameters of $\mathrm{La}_{8} \mathrm{Cu}_{7} \mathrm{O}_{19}$ determined from the best fit to the model associated with the $\Gamma_{2}$ irrep, with additional restrictions given in the main text. $\phi$ denotes the angle between a $\mathrm{Cu}$ moment and the $a$ axis; $\theta$ is the angle between the $\mathrm{Cu}$ moment and the $c$ axis.

\begin{tabular}{lcccc}
\hline \hline $\mathrm{La}_{8} \mathrm{Cu}_{7} \mathrm{O}_{19}$ & $T=10 \mathrm{~K}$ & Space group: & $C 2 / c$ & \\
Observed refl. & 179 & & & \\
$\chi^{2}$ & 6.5 & & & \\
\hline Site & $\begin{array}{c}\text { Moment } \\
\left(\mu_{B}\right)\end{array}$ & $\begin{array}{c}\mathrm{deg}) \\
(\mathrm{deg})\end{array}$ & \\
$\mathrm{e} 1$ & $0.69(7)$ & $90(0)$ & $90(0)$ & $\mathrm{e} 3$ \\
$\mathrm{e} 2$ & $0.69(7)$ & $180(0)$ & $90(0)$ & $\mathrm{e} 4$ \\
$\mathrm{f} 11$ & $0.73(7)$ & $0(0)$ & $90(0)$ & $\mathrm{f} 13$ \\
$\mathrm{f} 12$ & $0.73(7)$ & $0(0)$ & $90(0)$ & $\mathrm{f} 14$ \\
$\mathrm{f} 15$ & $0.73(7)$ & $270(0)$ & $90(0)$ & $\mathrm{f} 17$ \\
$\mathrm{f} 16$ & $0.73(7)$ & $90(0)$ & $90(0)$ & $\mathrm{f} 18$ \\
$\mathrm{f} 21$ & $1.17(5)$ & $190(10)$ & $90(8)$ & $\mathrm{f} 22$ \\
$\mathrm{f} 22$ & $1.17(5)$ & $145(20)$ & $27(9)$ & $\mathrm{f} 24$ \\
$\mathrm{f} 25$ & $1.17(5)$ & $57(10)$ & $78(8)$ & $\mathrm{f} 27$ \\
$\mathrm{f} 26$ & $1.17(5)$ & $275(11)$ & $95(7)$ & $\mathrm{f} 28$ \\
$\mathrm{f} 31$ & $1.32(6)$ & $279(7)$ & $81(8)$ & $\mathrm{f} 33$ \\
$\mathrm{f} 32$ & $1.32(6)$ & $75(7)$ & $119(8)$ & $\mathrm{f} 34$ \\
$\mathrm{f} 35$ & $1.32(6)$ & $240(6)$ & $97(7)$ & $\mathrm{f} 37$ \\
$\mathrm{f} 36$ & $1.32(6)$ & $117(7)$ & $96(9)$ & $\mathrm{f} 38$ \\
\hline \hline
\end{tabular}

In the next step, we have performed a series of more symmetrical fits, introducing more and more symmetry relations that are based either physically (equal moments on equivalent sites) or on previous fit results (some fitted parameters suggest a special direction of moments). Refined $\theta$ values for $\mathrm{Cu}$ moments in the octahedral sites (the e1...e4 and the f11..f 18 sites) suggest their orientation perpendicular to the $c$ axis. Fixing $\theta=90$ leads to a reduction of the free parameters to 38. Such a reduction is justified by the $\chi^{2}$ of 6.1 for this fit, which is only marginally worse than the general fit that takes into account the symmetry. Inspection of the refined parameters shows that it is possible to reduce the number of free parameters even further. Namely, moments in the e1...e4 and the f11..f 18 positions could be fixed along the $b$ axis or perpendicular to it, building two types of chains, reducing the number of free parameters to 30 . In this case, the $\chi^{2}$ increases to 6.2 . Finally, by assuming equal moments within each type of Wyckoff site that seems to be a reasonable physical assumption, one obtains parameters that are listed in Table III. The corresponding AF structure is shown in Fig. 6. The obtained $\chi^{2}$ factor of 6.5 for this fit with 20 free parameters is not much higher than values following from more general fits described above. The agreement between the observed and calculated magnetic structure factors squared is shown in Fig. 7.

Any other more symmetrical model (e.g., fixing the direction of moments in the $\mathrm{f} 21$...f 28 and/or f 31 . .f 38 sites) leads to a worse agreement with the data. For instance, fits with only a single free parameter (the moment magnitude), with all $\phi$ and $\theta$ values fixed to directions either parallel to the $a$ or $b$ axis lead to a $\chi^{2}$ factor of 12.7 and an average $\mathrm{Cu}$ moment of $0.85(7) \mu_{B}$. Crucial seems to be the noncollinearity of moments on the

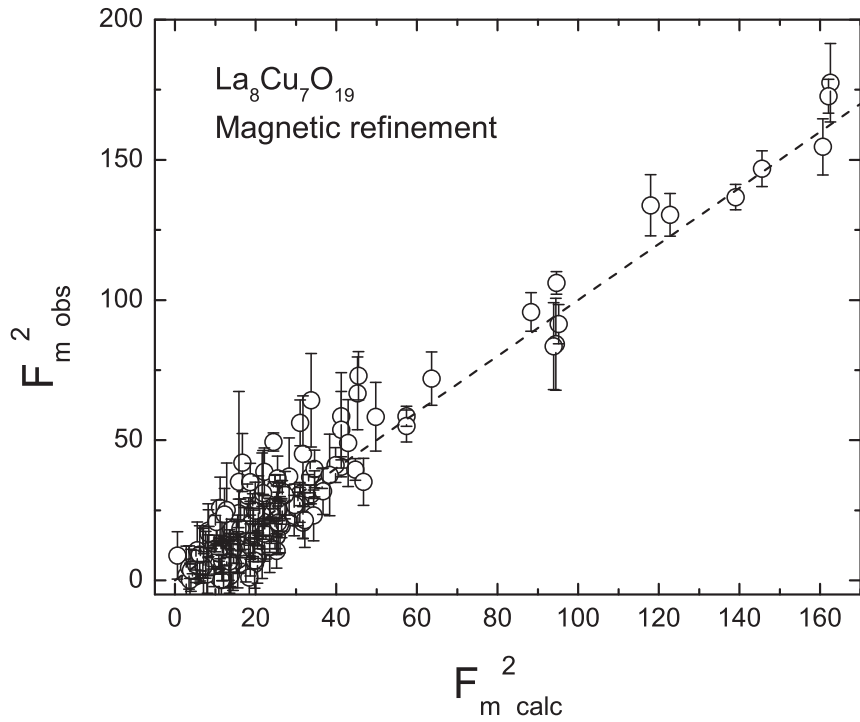

FIG. 7. Plot of the calculated versus observed squared magnetic structure factors collected on a $\mathrm{La}_{8} \mathrm{Cu}_{7} \mathrm{O}_{19}$ single crystal after correction for the extinction and Lorentz factor and refined using the model shown in Fig. 6.

tetrahedral sites. We conclude that the noncollinearity is a significant feature of the AF structure of $\mathrm{La}_{8} \mathrm{Cu}_{7} \mathrm{O}_{19}$. It is, however, not of the type suggested previously in the literature.

\section{DISCUSSION AND CONCLUSIONS}

Our results clearly suggest that $\mathrm{La}_{8} \mathrm{Cu}_{7} \mathrm{O}_{19}$ orders $\mathrm{AF}$ at low temperatures with two kinds of $\mathrm{Cu}$ moments divided into two subsystems coupled in a complicated noncollinear fashion. All moments are divided into pairs that are coupled ferromagnetically within the pairs (according to the representation $\Gamma_{2}$ ), in agreement with the symmetry analysis. Moment magnitudes vary between 0.7 and $1.3 \mu_{B}$.

The antiferromagnetic coupling of moments in the $4 \mathrm{e}$ and f11...f18 sites (octahedral positions), forming the rungs (as indicated in Fig. 6), is understandable from the GoodenoughKanamori rules [21-23]. These are based on a superexchange of an (virtual) electron between two neighboring cations through a nonmagnetic anion. If the coupling is mediated by the same $p$ orbital (the bonding angle is $180^{\circ}$ ), the exchange should be strongly antiferromagnetic. If different $p$ orbitals are involved or the bonding angle is close to $90^{\circ}$, the coupling is usually weak and ferromagnetic. In the case of $\mathrm{La}_{8} \mathrm{Cu}_{7} \mathrm{O}_{19}$ we note that $\mathrm{Cu}$ sites in rungs, for instance $\mathrm{Cu} 1$ (in Fig. 6 denoted as e2) and $\mathrm{Cu} 2$ (denoted as f12), are coupled via a link through an oxygen $\mathrm{O} 2$ (see Table I for coordinates, and Fig. 1) at a distance of $1.9 \AA$ that is from both $\mathrm{Cu}$ sites the same and which is very close to $180^{\circ}$. Another linear link, this time along the $b$ axis exists between $\mathrm{Cu} 1(\mathrm{Cu} 2)$ moments via $\mathrm{O} 1(\mathrm{O} 4)$ atoms, respectively. It is therefore to be expected that the $\mathrm{Cu} 1$ and $\mathrm{Cu} 2$ sites (4e and $\mathrm{f} 11 \ldots \mathrm{f} 18$ moments) are coupled strongly antiferromagnetically within the chains (rungs) and along the $b$ axis. This is indeed the case. Moreover, because the interatomic $\mathrm{Cu}-\mathrm{O}$ distances are in all cases roughly the same for these links such local moment 
arrangement is nearly two dimensional. In view of these facts, it remains puzzling that the analysis of the static susceptibility yields an unusual low $J / k_{B}=194(3) \mathrm{K}$, as mentioned already above.

The situation at both ends of the rungs is less clear. The moments residing in the $\mathrm{Cu} 3$ sites at the edge of the ladder rung (f21..f 28 sites-see Fig. 1) seem to have all three Cartesian components. However, these moments have still a clear tendency to keep predominantly directions as in the middle of the rungs (at the $4 \mathrm{e}$ and $\mathrm{f} 11$...f 18 sites). This finding is also not surprising as these moments are coupled via nearly linear $\mathrm{Cu} 3-\mathrm{O} 3-\mathrm{Cu} 2$ links on one side to octahedral $\mathrm{Cu} 2$ site moments that are directed along either $a$ or $b$ axis, and on the other side via $\mathrm{Cu} 3-\mathrm{O} 5-\mathrm{Cu} 4$ to nearly rectangular bonds with tetrahedral $\mathrm{f} 31 \ldots \mathrm{f} 38$ moments. The $\mathrm{O} 5$ atoms provide a connection between "rung" and tetrahedral "ribbon" sites, i.e., a coupling between rungs in the third dimension. The same $\mathrm{O} 5$ oxygen provides at the same time also a nearly $180^{\circ}$ bond along the $b$ axis between $\mathrm{Cu} 3$ sites. The deviation from the ideal value of $180^{\circ}$ is, however, much larger than in the case of the coupling of $\mathrm{Cu} 1$ and $\mathrm{Cu} 2$ type moments along this direction. $\mathrm{Cu} 3$ moments are therefore to a certain extent magnetically frustrated. Since the $90^{\circ}$ superexchange bonds are usually much weaker than the linear antiferromagnetic ones, the antiferromagnetic coupling of $\mathrm{Cu} 3$ moments to $\mathrm{Cu} 2$ moments (in linear rungs) prevails. At best, this is seen for instance in the case of the moment labeled in Fig. 6 as f21. Its major $a$ axis or $b$ axis components, depending on the chain, are antiferromagnetically coupled within the rungs. The coupling type within the tetrahedral $\mathrm{f} 31$...f 38 moment subsystems is not easy to predict using the Goodenough-Kanamori rules as there are, except for ferromagnetic near-rectangular bonds to $\mathrm{Cu} 3$ sites, neither $90^{\circ}$ nor $180^{\circ}$ links involving an oxygen atom. However, also in this case one can expect the moments to have all three Cartesian components.

Let us now turn to the $\beta$ parameter as determined from one of the most intense magnetic reflections in the close vicinity of $\mathrm{T}_{N}$. As mentioned above, our parameter $\beta=0.27(2)$ is larger than that of Zobkalo et al. $(\beta=0.13(2))$ [10]. It is interesting to note that both values are very close to boundary values of the universal window observed for critical exponents $(0.13$ to 0.23 ) in two-dimensional $X-Y$ systems [24]. While that one of Zobkalo et al. lies at the lower boundary, our value lies above the upper one, suggesting a crossover to a threedimensional behavior. Since $\mathrm{La}_{8} \mathrm{Cu}_{7} \mathrm{O}_{19}$ orders magnetically at rather high temperatures the application of a two-dimensional model seems to be not entirely adequate. On the other hand, a value $\beta=0.27(2)$ is somewhat lower than the values expected for a 3D antiferromagnet, but still substantially larger than a value of 0.125 expected for an Ising system [18].
In conclusion, the deduced AF structure of $\mathrm{La}_{8} \mathrm{Cu}_{7} \mathrm{O}_{19}$ is strongly noncollinear and in disagreement with the structure described by Zobkalo et al. [10]. An agreement between the two suggestions can be found only for one half of the $\mathrm{Cu}$ moments in octahedral sites $(\mathrm{e} 1, \ldots, \mathrm{e} 4$ and $\mathrm{f} 11, \ldots, \mathrm{f} 18)$, i.e., for moments in Chain II. In Chain I are $\mathrm{Cu}$ moments oriented perpendicular to Chain II. For $\mathrm{Cu}$ moments situated at the ends of the rungs (f21 to f28), and those situated in the ribbons ( $\mathrm{f} 31$ to $\mathrm{f} 38$ ), we find orientations that have all three Cartesian components. The experimentally determined coupling between the moments can be explained on the basis of the Goodenough-Kanamori rules.

Long range magnetic ordering of three-dimensional nature at a rather high temperature of $103 \mathrm{~K}$ clearly suggests the presence of significant interaction between ladder planes stacked along the [100] direction. The quasi-one-dimensional character of the model five-leg ladder is therefore realized by this compound only above the ordering temperature (as seen from the fits to the susceptibility data). The physics of such an isotropic spin ladder is expected to be qualitatively similar to that of a gapless $S=1 / 2$ chain [16]. However, it becomes difficult to verify the above owing to a threedimensionally ordered ground state. At high temperatures, the broad maximum in susceptibility beyond $\mathrm{T}_{N}$ indicates the presence of short-range order in the ladder planes. Measurements of spin-spin correlation lengths above the ordering temperature could provide more precise insight into the effective dimensionality of these short-range interactions in the spin ladder under consideration and on the nature and strength of exchange interactions between isolated ladders along the [101] and [100] directions [25,26]. These interactions seem to be complicated by the geometry near the rung edges, where the placements (and spin moment orientations) of $\mathrm{Cu}(3,4)$ and $\mathrm{O}$ atoms suggest multiple exchange paths and the presence of frustrated interactions between ladder units which possibly control the spin-spin correlation lengths along these directions and in turn, $T_{N}$. Investigations of the dispersion of spin waves in different directions at low temperatures could also provide more detailed information about these and additional interactions.

\section{ACKNOWLEDGMENTS}

K.P. acknowledges given CRG D23 beamtime at ILL. The work at IFW has been supported by the European Commission through the LOTHERM project (Project No. PITN-GA-2009238475), and the Deutsche Forschungsgemeinschaft (DFG) through SFB 1143, HE 3439/12, and WO 1532/3-2. We would like to thank R. Wimpory from HZB for carefully checking our paper.
[1] E. Dagotto, Rep. Prog. Phys. 62, 1525 (1999).

[2] A. N. Vasiliev, M. M. Markina, and E. A. Popova, Low Temp. Phys. 31, 203 (2005).

[3] D. C. Johnston, R. K. Kremer, M. Troyer, X. Wang, A. Klümper, S. L. Bud'ko, A. F. Panchula, and P. C. Canfield, Phys. Rev. B 61, 9558 (2000).
[4] E. Dagotto and R. M. Rice, Science 271, 618 (1996).

[5] M. Uehara, T. Nagata, J. Akimitsu, H. Takahashi, N. Môri, and K. Kinoshita, J. Phys. Soc. Jpn. 65, 2764 (1996).

[6] R. J. Cava, T. Siegrist, B. Hessen, J. J. Krajevski, W. F. Peck, Jr., B. Batlogg, H. Takagi, J. V. Waszczak, L. F. Schneemeyer, and H. W. Zandbergen, J. Solid State Chem. 94, 170 (1991). 
[7] B. Schüpp, C. Sekar, W. Gruner, G. Auffermann, C. Bähtz, and G. Krabbes, J. Anorg. Allg. Chem. 630, 663 (2004).

[8] Y. Zenitani, N. Watanabe, and J. Akimitsu, Physica C 341-348, 355 (2000).

[9] C. Sekar, T. Watanabe, A. Matsuda, H. Shibata, Y. Zenitani, and J. Akimitsu, J. Solid State Chem. 3156, 422 (2001).

[10] I. A. Zobkalo, V. A. Polyakov, O. P. Smirnov, S. V. Gavrilov, S. N. Barilo, D. I. Zhigunov, and M. Bonnet, Physica B 234-236, 734 (1997).

[11] A. Mohan, S. Singh, S. Partzsch, M. Zwiebler, J. Geck, S. Wurmehl, B. Büchner, and C. Hess, J. Cryst. Growth 448, 21 (2016).

[12] Oxygen atoms $(\mathrm{O} 7, \mathrm{O} 8)$ at $\approx 2.56 \AA$ and $2.62 \AA$ from the $\mathrm{Cu} 3$ site can be considered as apical atoms forming a highly distorted octahedral environment for the $\mathrm{Cu} 3$ atom. However, as these distances are rather large, $\mathrm{CuO}_{4}$ tetrahedral environments are assumed here.

[13] T. Roisnel and J. Rodriguez-Carvajal, Mater. Sci. Forum 378, 118 (2001).

[14] C. Sekar, B. Schüpp-Niewa, G. Krabbes, M. Wolf, D. Eckert, M. Knapp, and K.-H. Müller, J. Solid State Chem. 178, 28 (2005).
[15] D. C. Johnston, M. Troyer, S. Miyahara, D. Lidsky, K. Ueda, M. Azuma, Z. Hiroi, M. Takano, M. Isobe, Y. Ueda, M. A. Korotin, V. I. Anisimov, A. V. Mahajan, and L. L. Miller, arXiv:condmat/0001147.

[16] B. Frischmuth, B. Ammon, and M. Troyer, Phys. Rev. B 54, R3714 (1996).

[17] We mention that a similar analysis in terms of a $S=1 / 2$ chain model leads to unsatisfactory results, both in terms of the temperature dependence below $T_{\max }$ and the magnitude of $\chi$.

[18] R. J. Birgeneau, J. Skalyo, and G. Shirane, J. Appl. Phys. 41, 1303 (1970).

[19] A. D. Bruce, J. Phys. C: Solid State Phys. 14, 193 (1981).

[20] E. F. Bertaut, Acta Crystallogr. A 24, 217 (1968).

[21] J. B. Goodenough, J. Phys. Chem. Solids 6, 287 (1958).

[22] J. Kanamori, J. Phys. Chem. Solids 10, 87 (1959).

[23] J. Kanamori, J. Appl. Phys. Suppl. 31, 145 (1960).

[24] A. Taroni, S. T. Bramwell, and P. C. WHoldsworth, J. Phys.: Condens. Matter 20, 275233 (2008).

[25] K. R. Thurber, T. Imai, T. Saitoh, M. Azuma, M. Takano, and F. C. Chou, Phys. Rev. Lett. 84, 558 (2000).

[26] M. Greven, R. J. Birgeneau, and U.-J. Wiese, Phys. Rev. Lett. 77, 1865 (1996). 